VOL. I (1969), 269-278.

\title{
On the equivalence of invariant integrals and minimal ideals in semigroups
}

\section{N. A. Tserpes and A. G. Kartsatos}

Let $S$ be a Hausdorff topological semigroup and $C_{b}(S), C_{c}(S)$, the spaces of real valued continuous functions on $S$ which are respectively bounded and have compact support. A regular measure $m$ on $S$ is $x^{*}$-invarient if $m(B)=m\left(t_{x}^{-1}(B)\right)$ for every Borel $B \subset S$ and every $x \in S$, where $t_{x}: s \rightarrow s x$ is the right translation by $x$. The following theorem is proved: Let $S$ be locally compact metric with the $t_{x}$ 's closed. Then the following statements are equivalent: (i) $S$ admits a right invariant integral on $C_{c}(S)$. (ii) $S$ admits an $r^{*}$-invariant measure. (iii) $S$ has a unique minimal left ideal. The above equivalence is considered also for normal semigroups and analogous results are obtained for finitely additive $r^{*}$-invariant measures. Also in the case when $S$ is a complete separable metric semigroup with the $t_{x}$ 's closed, the following statements are equivalent: (i) $S$ admits a right invariant integral $I$ on $C_{b}(S)$ such that $I(1)=1$ and satisfying Daniel's condition. (ii) $S$ admits an $p^{*}$-invariant probability measure. (iii) $S$ has a right ideal which is a compact group and which is contained in a unique minimal left ideal. Finally, in order that a locally compact $S$ admit a right invariant measure, it suffices that $S$ contain a right ideal $F$ which is a left group such that $(B \cap F)_{x}=B X \cap F x$ for all Borel $B \subset S$.

Received 21 March 1969. Received by J. Austral. Math. Soc. 8 November 1968. Revised 19 February 1969. Communicated by G.B. Preston. The authors wish to express their indebtedness to the referee for his remarks. 


\section{Introduction}

In what follows $S$ will be at least a Hausdorff topological semigroup and $C_{b}(S), C_{c}(S)$, the spaces of all real valued continuous functions on $S$ which are bounded, have compact support respectively. For $x \in S, t_{x}$ denotes the right translation $s \rightarrow s x$ and for $B \subset S, B x^{-1}$ denotes the set $t_{x}^{-1}(B)=[s ; s x \in B]$. By a right (left) ideal of $S$ is meant a nonempty subset $A \subset S$ such that $A S \subset A(S A \subset A) . S$ is called a left group if $S$ is left simple (i.e., $S x=S$ for every $x \in S$ ) and contains an idempotent element; equivalently $S$ is a left group if $S$ is homeomorphic to a direct product $E \times G$ where $G=q S, q$ being any (fixed) idempotent of $S$, is a group, and $E$ is the set of idempotents of $S$. For example, consider the complex numbers $\neq 0$ with multiplication $x * y=x|y|$.

In case $S$ is locally compact then $C_{c}(S) \neq[0]$ and by an integral on $S$ we mean a nontrivial positive linear functional $I$ on $C_{c}(S)$. Given such a functional $I$ on $C_{c}(S)$, an integral can be defined on $C_{b}(S)$ by $\bar{I}(\phi)=\sup \left[I(\psi) ; \psi \in C_{c}(S), \psi \leqq \phi\right]$ for $\phi \in C_{b}^{+}(S)$, that is, $\bar{I}$ is the usual "canonical extension" of an integral from $C_{c}(S)$ to $C_{b}(S)$. [See 6 , p. 12l]. In the non-locally compact case by an integral on $S$ we will always mean a nontrivial non-negative linear functional $I$ on $C_{b}(S)$. In general $I$ would have to satisfy additional conditions in order to have a representation with respect to a measure. The support of an integral $I$ on $c_{b}$ is defined as the set of all $x \in S$ such that for every open $U \supset x$ there is $f \in C_{b}, 0 \leqq f \leqq 1, f=0$ on $S-U$, and $I(f) \neq 0$. We denote by $B(S)$ [resp. $B(S)$ ] the Borel algebra [resp. o-algebra] generated by all open sets of $S$. Unless otherwise specified, $m$ will denote a measure, i.e., $m$ is countably additive on $B(S) . m$ is right invariant if $m(B)=\bar{m}(B x)$ for every $B \in B(S)$ and every $x \in S$, where $\bar{m}$ is the completion of $m$ relative to $B(S)$. (Note that the definition includes

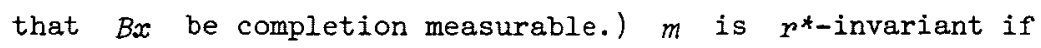
$m(B)=m\left(B x^{-1}\right)$ for every $B \in B$ and every $x \in S$. The support of $m$, always denoted by $F$, is defined as the set of all $x \in S$, every open 
neighborhood of $x$ has positive measure. $m$ is regular if $m$ is finite on compact sets, $m$ is outer regular [i.e., $m(B), B \in B$, is the inf of the measures of its open supersets], and $m(U)=\sup [m(C) ; C=$ compact $\subset U]$ for all open $U \subset S . m$ is weakly regular if $m$ is outer regular and $m(B)=\sup [m(K) ; K=$ closed $\subset B]$ for every $B \in B . m$ is a probability measure if $m(S)=1$. An integral $I$ on a locally compact $S$ is right invariant if $I(f)=\bar{I}\left(f \circ t_{x}\right) \equiv \bar{I}\left(f_{x}\right)$, for every $f \in C_{c}(S)$ and every $x \in S$. In the non-locally compact case an integral $I$ on $C_{b}(S)$ is right invariant if $I(f)=I\left(f_{x}\right)$ for every $f \in C_{b}(S)$ and every $x \in S$. In many of our theorems we will require that $S$ be complete, i.e., that $S$ admit a uniformity compatible with its topology with respect to which $S$ is a complete uniform space. Metric, paracompact, realcompact [3b, pp. 114, 226; called Q-spaces by Hewit+] spaces are complete in the above sense.

Argabright [2] proved the following result:

THEOREM 1.1. Let $S$ be locally compact and satisfy (\#) $C x^{-1}$ is compact for every $x \in S$ and every compact $C \subset S$. Then the following statements are equivalent:

(i) There is a right invariant integral $I$ on $C_{c}(S)$.

(ii) There is an $r^{*}$-invariant regular measure $m$ on $S$. (iii) $S$ has a unique minimal left ideal.

In this paper we consider the above equivalence for normal or locally compact metric semigroups without the compactness condition (\#). It turns out that if $S$ is metric then (H) can be replaced by a weaker condition, i.e., by

$$
\text { All right translations } t_{x}, x \in S \text {, are closed maps. }
$$

Substantial use of $(C)$ in connection with invariant integrals was made in [7, p. 186] and it was implied by the conditions used in a related work in [8]. Using a characterization of $r^{*}$-invariant measures on left groups given in [2, p. 378] we give a sufficient condition for the existence of a regular right invariant measure (integral) on a locally compact semigroup. 


\section{Some useful lemmata}

LEMMA 2.1. ([2]) Each right invariant integral I (resp. each $r^{*}$-invariant measure $m$ ) on a locally compact left group $S=E \times q S$ is given by $I(f)=\int_{S} f(x) d\left(m_{1} \times m_{2}\right)$, where $m_{1}=$ a Borel measure on $E$ and $m_{2}=$ a right Haar measure on the locally compact group qS resp. $m=m_{1} \times m_{2}$, which is also right invariant on $S$, the mappings $t_{x}$ on left groups being homeomomphisms). Moreover if $m$ is $r^{*}$-invariant on a Hausdorff semigroup $S$ and $m(S-F)=0$, then $F$ is a right ideal of $S$ and $F x=F$ for all $x \in S . \quad[\overline{F x}=$ the closure of $F x$ in $S]$.

LEMMA 2.2. The closure of a left subgroup $F$ of $S$ which is also a right ideal of $S$ is also a left subgroup.

Proof. By continuity, every right identity for $F$ is also a right identity for $\bar{F}$; for if $x_{\beta} \rightarrow z \in \bar{F}, x_{\beta} \in F$, and $e$ is an idempotent in $F$ ( $e$ is also a right identity), then $x_{\beta} e \rightarrow z e$, so that $z e=z$. Hence for $a \in F$, there is $c \in F$ such that $c a=e$ and so $\bar{F} a \supset \bar{F} c a=\bar{F}$. Next for $z \in \bar{F}, \bar{F} z \supset \bar{F} f z, f$ being any element of $F$, and $f z \in F$, so that $\bar{F} z \supset \bar{F}$.

That condition (\#) implies condition (C) is shown by the following

LEMMA 2.3. Let $f: X \rightarrow Y$ be any map, $X, Y$ Hausdorff spaces; then

(i) $f$ closed and $f^{-1}(y)=$ compact, for alz $y \in Y$, implies

(ii) $f^{-1}(C)=$ compact, for all compact $C \subset Y$.

The proof of this lema (formulated in different terminology) is essentially contained in N. Bourbaki [3a, pp. 101, 104 and p. 37, Prop. 3]. As an example, consider $S=[0,1 / 2)$ with ordinary multiplication and usual topology; clearly the $t_{x}$ are closed but $[0] 0^{-1} .=S=$ not compact.

3. Theorems on invariant integrals and measures

The following theorem has an immediate application to locally compact metric semigroups satisfying (C).

THEOREM 3.1. Let $S$ be zocally compact, normal, $1^{\text {st }}$-countable, 
satisfying (C). Then the following statements are equivalent:

(i) There is a right invariant integral $I$ on $C_{c}(S)$.

(ii) There is a regular $r^{*}$-invariant Borel measure $m$ on $S$.

(iii) $S$ has a closed right ideal $E$ which is a left group (and which is necessarily contained in a closed unique minimal left ideal $L$ of $S$ ).

Proof. The proof that $(i) \Rightarrow(i i)$ is the same as in [2, p. 379]. To prove that $(i i) \Rightarrow$ (iii) we only need to show that $F$ has an idempotent, since by (C) and Lemma 2.1, $F$ is left simple and a right ideal. For if $F$ is shown to be a left group, then $L=\bigcap_{x \in S} S x \supset F$ and by Lemma 3.2 , $\bar{L}=L . \quad[L$ is the unique minimal left ideal and also a right ideal. $]$ Let $A=a a^{-1} \cap F=[x \in F ; x a=a]$, for $a \in F ; A \neq \phi$ since the $t_{a}{ }^{\prime}$ s on $F$ are "onto" and $F$ is left simple. If $\operatorname{Int} A=\operatorname{Interior}(A) \neq \phi$ then by $r^{*}$-invariance, $m[a]>0$ and every $z \in a F$ is such that $m[z] \geqq m[a]$; (note that for $K=$ closed, $K x x^{-1} \supset K$ and so $m(K x) \geqq m(k)$ ); hence, if $z \in y y^{-1} \cap a F \neq \phi \quad(\alpha F$ is also left simple $), y \in a F$, then $\left[z, z^{2}, z^{3}, \ldots\right]$ is finite because $m\left[y y^{-1}\right]=m[y]>0$ and $m[y] \cdot<\infty$. Since every finite semigroup has an idempotent, $F$ is a left group. In case Int $A=\phi$, the boundary of $A$ is countably compact by [9, p. 10] and since $A$ is complete, $A$ is a compact semigroup and hence it contains at least one idempotent.. (See $[36]$, p. 237.)

Next we show that $(i i i) \Rightarrow(i)$; by Lemma $3.1, F$ admits an $r^{*}$-invariant measure $m=m_{1} \times m_{2}$ which can be extended to the whole $S$ as in $[2$, p. 380].

The following theorem has an immediate application to separable metric semigroups satisfying (C).

THEOREM 3.2. Let $S$ be normal, $1^{\text {st }}$-countable, realcompact, satisfying $(\mathrm{C})$. Then $(i) \Leftrightarrow(i i) \Rightarrow$ (iii), where

(i) There is a right invariant integral $I$ on $C_{b}(S)$ such that $I(1)=1$ and $I\left(f_{n}\right) \downarrow 0$ whenever $f_{n} \downarrow 0, f_{n} \in C_{b}(S)$. 
(ii) There is an $r^{*}$-invariant weakly regular probability measure $m$ on $S$.

(iii) $S$ has a closed right ideal $F$ which is a left group (and which is necessarity contained in a closed unique minimal left ideal L) .

Proof. (i) $\Rightarrow$ (ii): There is a weakly regular probability $m$ such that $I(f)=\int f(x) m(d x)$, for $f \in C_{b}(S) . \quad$ (see $[10$, p. 63]). (Actually $m$ is a Baire measure, i.e., $m$ is defined on the $\sigma$-field generated by the closed $G_{\delta}$ 's and then it is extended to a Borel measure by $m(B)=\sup \left[m(K) ; K=\right.$ closed $\left.G_{\delta} \subset B\right]$ as in $\left.[-3, \mathrm{pp} .183,203,194].\right)$

We can prove that $m$ is $r^{*}$-invariant either by using an argument in [2, p. 379] replacing local compactness and compact sets by normality and closed sets respectively, or, a method of Markov [7] who studied finitely additive outer probability measures on normal spaces.

That $(i i) \Rightarrow$ (iii) follows as in Theorem 3.1 provided that we can show that such a support $F$ for $m$ exists. Since $S$ is realcompact, the Baire restriction of $m$ has a support $F=$ (the intersection of all closed $G_{\delta}^{\prime}$ 's of measure 1). (See [1, p. 197] and [5, pp. 172-173]). [In particular if $S$ is separable metric and $m$ is a probability measure on $S$, then $F \neq \phi$ and $m(S-F)=0$. See $[12$, p. 27].]

COROLLARY 3.3. Let $S$ be a complete separable metric semigroup with property (c). Then each of the statements (i), (ii) of Theorem 3.2 is equivalent to the statement

(iii) $S$ has a right ideal $F$ which is a compact group (and which is necessarily contained in a closed unique minimal left ideal L) .

Proof. We show $(i i) \Rightarrow(i i i)$. As in the proof of Theorem 3.1, $F$ is a left group and $F=E \times q F$, where $E$ is the set of idempotents of $F$ and $q \in F$. Since $q F S=q F q S \subset q F F \subset q F, q F$ is also a right ideal of $S$. We show next that $q F$ is a compact group. Since $F=E \times G$, where $G$ is a group, by using first compact rectangles and the fact that $A B^{-1}=[x ; x b \in A$ for some $b \in B]$ is closed whenever $A, B \subset F$ are 
compact, one easily verifies that $A B^{-1}$ is compact. Let $C$ be compact $\subset F$ such that $m\left(x^{-1} C\right)>0$ for some $x \in F$. Such a $C$ exists because the measure $\bar{m}(A)=m\left(x^{-1} A\right)$ is regular, since $m$ is regular by [12, p. 29]. The proof of this fact given in [4, p. 179] is valid in our case. The function $m\left(x^{-1} C\right)$ is upper semi-continuous because $\left[x \in S ; m\left(x^{-1}(S-C)\right)>\alpha\right]$ is open as in [4, p. 179] whose proof carries over to the present case. Let $\varepsilon>0$ and $K$ compact $\subset F$ such that $m(K)>1-\varepsilon$; then for $x \notin C K^{-1}, m\left(x^{-1} C\right)<\varepsilon$ so that $f(x)=m\left(x^{-1} C\right)$ vanishes at infinity. Let $a \in F$ such that $f(a)=\sup _{x \in F} f(x)$. Now $f(a)-f(a x) \geqq 0$ and also $\int_{F}[f(a)-f(a x)] m(d x)=0$ because $m$ is an idempotent measure on $F$. (See $[12, p .67]$.) Since $[x ; f(a)-f(a x)>0]$ is open, it must be empty. Hence $f(a)=f(a x)$ for all $x \in F$, so that $\overline{a F} \subset M=\left[x \in F ; f(x)=\sup _{s \in S} f(s)\right]$ and so $\overline{a F}$ is compact. Now $\overline{a F}$ being a compact semigroup has an idempotent $e$ and therefore $e F=\overline{e F} \subset M$ and $e F$ is compact group. $(i i i) \Rightarrow(i)$. The Haar measure on $e F$ may be extended over the whole $S$ as in [2, pp. 380-381].

THEOREM 3.4. Let $S$ be a complete metric semigroup satisfying (C) and let the set of its idempotents be discrete in the relative topology. Then the following statements are equivalent:

(i) There is a continuous $r^{*}$-invariant Borel measure $m$ on $S$ with non-empty support. ( $m$ is called continuous if $m[x]=0$ for alz $x \in S$.

(ii) $S$ has a perfect right ideal $F$ which is a left group (and which is necessarily contained in a closed unique minimal left ideall.

Proof. (i) $\Rightarrow(i i)$ as in Theorem 3.1. Next we show (ii) $\Rightarrow(i)$ : Let $F=E \times q F ;$ since $F$ has no isolated points, $q F$ is dense-in-itself and a complete metric group. By [11, Th. 1.2], $q F$ admits a right invariant continuous (infinite) Borel measure $m_{2}$. By taking $m_{1}=$ a probability on $E, m=m_{1} \times m_{2}$ is $r^{*}$-invariant on $F$ and it can be extended to the whole $S$. (Note that $r^{*}$-invariance and right invariance coincide on left groups.) 
THEOREM 3.5. (Sufficient condition for existence of right invariant measure.) Let $S$ be locally compact. Then, in order that $S$ admit a right invariant regular Borel measure it suffices that $S$ contain a right ideal $F$ which is a left group and such that

(1) $(B \cap F) x=B x \cap F x$ for every $x \in S$ and every Borel $B \subset S$.

REMARK. Note that ( 1 ) is completely algebraic in nature, for if it holds for Borel $B$, then it holds for any $B \subset S$. In fact, we may consider $\bar{B}$ and then easily prove that it holds for $B$, since $F$ and $\bar{F}$ are left groups by Lemma 3.2 .

Proof. Since $\bar{F}$ is a left group, Lemma 2.1 gives a right invariant measure $m=m_{1} \times m_{2}$ on $\bar{F}$, and by defining $m^{*}(B)=m(B \cap \bar{F})$ for $B=$ Borel $\subset S$, one easily proves that $m^{*}$ is $r^{*}$-invariant on $S[2$, p. 380]. Now for $B=$ Borel $\subset S$, $m^{*}(B)=m^{*}(B \cap \bar{F})=m[(B \cap \bar{F}) q x]=m[(B \cap \bar{F}) x]=m(B x \cap \bar{F} x)=m(B x \cap \bar{F})=\bar{m}^{*}(B x)$, where $x \in S$ and $q$ is any idempotent (and right identity) of $\bar{F}$. Note that since $(B \cap F) x=B x \cap F x=B x \cap F$, we also have $(B \cap \bar{F}) x=B x \cap \bar{F} x=B x \cap \bar{F}$, since the mappings $t_{x}$ are homeomorphisms on $F$ and $\vec{F}$.

\section{Finitely additive measures}

As it was mentioned earlier an integral $I$ on $C_{b}(S)$ may not correspond to a Borel measure. However in many cases it is possible to find a finitely additive measure $m$ whose domain of definition includes $B(S)$ and which corresponds to the given functional $I$ in the sense that $I$ can be represented in terms of $m$ as in [1, pp. 180-183]. A finitely additive probability measure $m$ is a non-negative finitely additive set function on $B(S)$ (= the Borel algebra of $S$ ) with $m(S)=1$. The definition of regularity and of the support of $m$ are the same as those given for a Borel measure in Section 1 .

THEOREM 4.1. Let $S$ be normal, $1^{\text {st }}$-countable, complete, satisfying (c). Then $(i) \Longleftrightarrow(i i) \Rightarrow$ (iii), where

(i) There is a right invariant integral $I$ on $C_{b}(S)$ with $I(1)=1$ and with nonempty support. 
(ii) There is an $r^{*}$-invariant weakly regular finitely additive probability measure on $S$ with nonempty support.

(iii) $S$ has a closed right ideal which is a left group (and which is necessarily contained in a closed unique minimal left ideal of S).

Proof. (i) $\Leftrightarrow$ (ii) follows as in [7]. (ii) $\Rightarrow$ (iii) as in Theorem 3.1. It is not known if $(i i i) \Rightarrow(i)$ even when $S$ is a separable metric semigroup.

\section{References}

[1] L. Argabright, "Invariant means on topological semigroups", Pacific J. Math. 16 (1966), 193-203.

[2] L. Argabright, "A note on invariant integrals on locally compact semigroups", Proc. Amer. Math. Soc. 17 (1966), 377-382.

[3] S.K. Berberian, Measure and Integration, (MacMillan Co., New York, 1965).

[3a] Nicolas Bourbaki, General Topology, Part 1, (Addison-Wesley, Reading, Mento Park, London, Ontario, 1966).

[36] Leonard Gillman and Meyer Jerison, Rings of continuous functions, (Van Nostrand, Princeton, 1960).

[4] M. Heble and M. Rosenblatt, "Idempotent measures on a compact topological semigroup", Proc. Amer. Math. Soc. 14 (1963), 177-184.

[5] Edwin Hewitt, "Linear functionals on spaces of continuous functions", Fund. Math. 37 (1950), 161-189.

[6] Edwin Hewitt and Kenneth A. Ross, Abstract hamonic analysis (Academic Press, New York, London, 1963).

[7] A. Markov, "On mean values and exterior densities", Rec. math. Moscou (Mat. Sb.) 4 (1938), 165-190.

[8] J.H. Michael, "Right invariant integrals on locally compact semigroups", J. Austral. Math. Soc. 4 (1964), 273-286.

[9] Ki iti Morita and Satirọ Hanai, "Closed mappings and metric spaces", Proc. Japan Acad. 32 (1956), 10-14. 
[10] J. Neveu, Mathematical foundations of the calculus of probabilities (Holden-Day Inc., San Francisco, 1965).

[11] John C. Oxtoby, "Invariant measures in groups which are not locally compact", Trans. Amer. Math. Soc. 60 (1946), 215-237.

[12] K.R. Parthasarathy, Probability measures on metric spaces (Academic Press, New York, London, 1967).

[13] G.B. Preston, "Inverse semigroups with minimal right ideals", $J$. London Math. Soc. 29 (1954), 411-419.

Department of Mathematics,

University of South Florida,

Tampa, Florida, USA.

Department of Mathematics,

University of Athens,

Athens, Greece. 